\title{
Drug resistance in cancer - searching for mechanisms, markers and therapeutic agents
}

\section{Robert O'Connor PhD, Martin Clynes PhD, Paul Dowling PhD, Norma O'Donovan PhD \& Lorraine O'Driscoll PhD}

To cite this article: Robert O'Connor PhD, Martin Clynes PhD, Paul Dowling PhD, Norma O'Donovan PhD \& Lorraine O'Driscoll PhD (2007) Drug resistance in cancer - searching for mechanisms, markers and therapeutic agents, Expert Opinion on Drug Metabolism \&

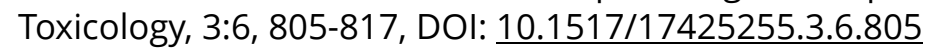

To link to this article: http://dx.doi.org/10.1517/17425255.3.6.805

曲 Published online: 21 Nov 2007.

Submit your article to this journal $ऍ$

Џlll Article views: 153

Q View related articles $\sqsubset$ 


\section{Oppert}

1. Introduction

2. Drug efflux pumps

3. Emerging role of drug uptake transporters

4. Targeted therapies and resistance

5. Resistance due to stromal factors

6. Proteomic analysis of drug resistance in cancer

7. RNA expression patterns and their manipulation in drug resistance

8. Conclusion

9. Expert opinion

\section{informa}

healthcare

\section{Drug resistance in} cancer - searching for mechanisms, markers and therapeutic agents

\author{
Robert O’Connor, Martin Clynes ${ }^{\dagger}$, Paul Dowling, Norma O’Donovan \& \\ Lorraine O'Driscoll \\ Dublin City University, National Institute for Cellular Biotechnology (NICB), Dublin 9, Ireland
}

Treatment resistance, whether inherent or acquired, is a major problem reducing the activity of conventional and newer, molecularly targeted, cancer drugs. A more complex picture of the causes and contributions of specific forms of resistance is now emerging through application of pharmacological, proteomic and gene expression technologies and we have entered an exciting time where new molecular research tools are being applied not only to characterise the causes of such resistance, but to identify rational new treatments and treatment combinations that are being rapidly translated to clinical evaluations with increasing success. This review outlines many of the contributing causes of resistance to established cytotoxics and to the new breed of molecularly targeted agents, both monoclonal antibodies and small molecules, and the research methods being used to wage war on resistant cancer.

Keywords: cancer chemotherapy, drug resistance, multiple drug resistance, targeted therapy, tyrosine kinase

Expert Opin. Drug Metab. Toxicol. (2007) 3(6):805-817

\section{Introduction}

Chemotherapy, including new molecularly targeted therapies, makes a significant contribution to the treatment of cancer patients. Major limitations to chemotherapeutic effectiveness arise from the toxic effects of these agents on normal cells and from resistance - inherent or acquired - to therapeutic agents. Combination chemotherapy was devised to overcome resistance, by treating with agents that exert their effects by different mechanisms and/or are very different chemically. Cancer cell populations can, unfortunately, respond by becoming multi-drug resistant (MDR) to a panel of mechanistically and structurally diverse drugs.

The presence of primitive stem cell populations within the the tumour mass combined with the tumour's ability to generate a wide range of variants, due to genomic instability associated with defective damage detection and repair and high proliferation rate, is characteristic of many cancers [1]. Resistant and MDR variants inherently present or generated may then be selected, in a Darwinian fashion, by multiple cycles of chemotherapy; of course many chemotherapeutic agents are mutagenic, thus increasing the frequency of resistant mutants in the cancer cell population.

Molecular mechanisms of drug resistance include overexpression of drug efflux pumps (generally ATP-binding cassette $[\mathrm{ABC}]$ transporter family members), such as the $m d r-1$ gene product $\mathrm{P}$-glycoprotein (P-gp)/P-170 (ABCB1), multi-drug resistant associated protein-1 (MRP-1; $\mathrm{ABCC} 1$ ) and related proteins, and breast cancer resistance protein (BCRP; ABCG2). It has also been suggested that drug 
sequestration [2-4], possibly with some involvement of vault proteins (e.g., major vault protein/lung resistance-related protein), could play a role in drug resistance. Important also are the relative levels of pro- and antiapoptotic proteins; altered levels and activities of target enzymes such as topoisomerase II (for anthracyclines), dihydrofolate reductase (for methotrexate), thymidylate synthetase (for various antimetabolites); and altered levels of drug-degrading enzymes, including glutathione-S-transferases and possibly some CYPs [1].

A major challenge is that drug resistance can be multifactorial, with different mechanisms being coexpressed within the same cell, and different subpopulations within the tumour expressing different sets and extents of resistance mechanisms [5]. Resistance can also relate to physical and physiological parameters such as $\mathrm{pH}$, oxygen tension, degree of tumour vascularisation, various aspects of the tumour microenvironment including extracellular matrix composition, tumour architecture and so on, so that individual changes in the cellular and molecular biology of the tumour cells may not always fully account for the observed resistance phenotype.

Nevertheless, delivery of adequate drug dose to the (usually) intracellular target is a necessary, even if not always sufficient, part of successful chemotherapy. Much resistancerelated research, therefore, both laboratory and clinical, has focused on reversing or circumventing resistance by inhibiting the activity of the efflux pumps [6], or by treating resistant cells/tumours with drugs that are not substrates for the pump being overexpressed.

\section{Drug efflux pumps}

Despite the diverse array of genetic and molecular changes that can take place in various cellular drug targets and response intermediates, the fundamentals of whether a drug can reach a relevant target concentration, that is, cellular drug pharmacokinetics, remains of key importance to the life and death of a tumour cell [7].

A major advance in our understanding of resistance mechanisms emerged in 1976 with the description of the MDR-1 (P-gp) protein and its characterisation as a cellular efflux pump [8].

P-gp (MDR-1, and now termed ABCB1) exists as a large transmembrane protein that actively moves a large number of xenobiotics, including many cytotoxic cancer drugs, from one leaflet of the plasma membrane to the other [9]. In recent years, several of the newer molecularly targeted cancer drugs such as tyrosine kinase inhibitors have also been shown to be active substrates for this pump and this may explain how some cancer cells can become resistant to these drugs [10]. A second cellular pump, MRP-1 (ABCC1), also pumps many of the important cytotoxic chemotherapy drugs [11]. BCRP (MXR, ABCG2) is a smaller transmembrane protein, which unlike the other major drug transporters, dimerises to generate a functional transporter unit [12].
Despite significant functional, physiological, mechanistic and structural differences between them, P-gp, MRP-1 and BCRP have a significant overlap in substrate and inhibitor specificity. The majority of the commonly used chemotherapy drugs, for example, taxanes, anthracyclines and vinca alkaloids, as well as newer molecularly targeted agents, are substrates for one or more of these pumps. In human cells, the largest family of drug efflux pumps are from the $A B C C$ protein family, that is, proteins with a close sequence homology with the MRP-1 pump, with nine constituent proteins members described [13]. Of the ABCC family, MRP-1 has shown the greatest association with drug resistance in a broad range of human cancers; other members of this protein family appear to have roles in resistance to a narrower more structurally distinct range of drugs. For example, overexpression of MRP-4 (ABCC4) and MRP-5 (ABCC5) have shown to confer resistance to certain nucleotide analogues used in treatment of some forms of leukaemia, although recent data may cast doubt on the physiological relevance of this activity [14]. The roles of other MRP-family proteins appear to be purely physiological.

These transporters use the energy of ATP conversion by highly conserved $\mathrm{ABC}$ motifs to drive the translocation of substrate drugs from one part of the lipid bilayer to another against a concentration gradient [15]. The mechanism of this action has been best characterised for P-gp, with the ATPase activity physically moving a drug binding pocket from one part of the protein structure to another while simultaneously decreasing the drug affinity of this pocket [16]. Despite some similarities in substrate specificity, MRP-1 and MRP-2 pumps appear to have a very different mechanism of action from P-gp and require the presence of a cotransport glutathione molecule either attached to the target agent or available in sufficient free concentration within the cell [17].

All of the known resistance transporters have important physiological functions in normal cells and organs: to move xenobiotics in a specific direction within a tissue, to protect cells/organs/the whole organism from toxicant action and/or to concentrate metabolic products for elimination [18]. However, increased expression/activity in resistant cancer can lead to a role in protecting the tumour cell from the effects of chemotherapeutic agents. Numerous studies have shown the prognostic relevance of specific transporters in particular types of cancer [19]. These findings are by no means uniform and complicating interpretive factors include the techniques used to study transporter expression, the potential for post-translational modifications, which affect transporter stability and activity, and polymorphisms, which can modify the identification of the transporter and its substrate specificity [20,21]. Nevertheless, the sheer weight of genetic, protein and functional studies indicates a significant role for $A B C$ transporters in cancer resistance $[1,18]$. 


\subsection{Circumvention of cancer efflux pumps}

Attempts have been made to use drug pump inhibitors to circumvent resistance. Agents that inhibit cancer resistance mechanisms are termed resistance modulators, chemosensitising agents or resistance circumvention agents. Although it has been possible to enhance cellular sensitivity to these drugs in vitro by pretreating the cells with other non-cytotoxic P-gp substrates, including verapamil, ciclosporin and more specific agents such as tariquidar, randomised clinical trials have largely failed to demonstrate superior clinical outcomes for this approach compared with chemotherapy alone [22-24]. These therapeutic failures are the result of the complex pharmacology of P-gp inhibition in human beings and the critical, body-wide protective role against xenobiotic action played by this pump. One of the major findings of combination studies of conventional cytotoxic P-gp chemotherapy agents, with even the latest generations of P-gp inhibitors, is that the inhibitor reduces substrate clearance, generating higher circulating levels and thereby producing no measurable increase in antitumour selectivity. Stated simply, although higher drug concentrations may be generated in the tumour through inhibition of P-gp, higher levels are also evident in the body, thus increasing general side effects and toxicity and often requiring chemotherapy dose reduction. Clinical findings are often similar to those that might be expected if patients had received higher chemotherapeutic drug doses. Clearly, therefore, effective clinical application of P-gp inhibition strategies to improve chemotherapeutic efficacy will rely on technologies that specifically target tumour P-gp without alterating the activity of the non-tumour pump [25].

Very few clinical circumvention studies have been conducted to combat the actions of other MDR pumps [6], but the lessons of P-gp inhibitor trials suggest that effective use of such therapies will require better patient stratification based on in vivo characterisation of real pump activity levels.

\section{Emerging role of drug uptake transporters}

To be effluxed by a drug transporter, the chemotherapeutic agent must clearly enter the cancer cell in the first place. Some drugs may enter the cell by passive diffusion and related nonspecific transport mechanisms; in the last 5 years, however, a number of pumps/transporters have been identified that appear to play a significant role in uptake of specific cancer drugs in cells [26,27]. These uptake proteins belong to the solute carrier family and include a number of organic anion transporter proteins (OATPs). Proteins such as OATP1B3 (OATP8) have been shown to play a important role in the uptake of taxane chemotherapy drugs [27]. In the case of cisplatin, a copper transporting pump has been demonstrated to play a significant role in the uptake of this drug in cells with downregulation of the pump, leading to reduced drug toxicity [28]. Because of the novelty of this class of transporters, there is a significant deficit in our understanding of their role in cancer resistance [26], but a number of tantalising observations have been made, such as the impact of polymorphisms of these proteins in drug pharmacology. Evidence already exists demonstrating a complex cellular coordination of uptake and efflux proteins in cells [29], and a recent study has demonstrated downregulation of the OATP8 (taxane transporter) in specific forms of cancer but not others [30].

\section{Targeted therapies and resistance}

In recent years the development of new anticancer treatments has shifted from cytotoxic agents to the development of molecularly targeted agents based on laboratory investigations of cancer cell biology. These novel therapies target key signalling proteins (such as tyrosine kinases) that are specifically altered in cancer cells and in so doing have fewer side effects than conventional cytotoxics drugs. For example, one of the first successful small-molecule inhibitors, imatinib, inactivates the kinase activity of the BCR-ABL fusion protein in chronic myeloid leukaemia (CML). At 18 months a complete cytogenetic response rate of $74 \%$ has been achieved in CML patients in response to imatinib treatment [31]. However, despite the early successes with imatinib, acquired resistance emerged quickly and mechanisms of resistance to imatinib are now well understood. Several other targeted therapies are now approved for the treatment of specific tumour types and a large number of potential therapies are in preclinical and clinical development. These targeted therapies can be divided into two main categories: small-molecule inhibitors and monoclonal antibodies.

\subsection{EGF receptor family (ErbB-1 and -2) inhibitors}

Trastuzumab is a monoclonal antibody that binds an external epitope of the HER-2 (ErbB-2) receptor and is approved for the treatment of both metastatic and early stage HER-2-positive breast cancer [32,33]. Lapatinib, a smallmolecule tyrosine kinase inhibitor of HER-2 and EGF receptor (EGFR), is in clinical development and has shown very promising results as a single agent and in combination with capcitabine [34]. A number of agents that target EGFR (ErbB-1) are either approved or are being tested in clinical trials for particular cancer types. Among the anti-EGFR monoclonal antibodies, cetuximab is the furthest ahead in clinical development. Cetuximab binds to EGFR with high affinity, competes with ligand binding and blocks activation of receptor tyrosine kinase by EGF or TGF. Gefitinib is an oral, selective and reversible smallmolecule inhibitor of EGFR that has mainly been investigated in non-small-cell lung cancer (NSCLC). There is evidence of a lack of correlation between EGFR expression levels in tumours and response to EGFR inhibition; this was confirmed by analysis of tumour samples from the gefitinib single-agent Phase II study in NSCLC. Two independent 
groups subsequently identified mutations in the EGFR gene, in a subgroup of NSCLCs, which correlate with clinical response to gefitinib [35,36]. The mutations lead to increased growth factor signalling and confer susceptibility to gefitinib. Erlotinib is also an EGFR-specific quinazoline derivative and has demonstrated benefit for NSCLC patients who have relapsed on standard chemotherapy.

\subsubsection{Angiogenesis inhibitors}

Several therapeutic strategies have been developed to inhibit angiogenesis in tumours, with the aim of starving the tumours of essential nutrients required for growth. The key targets for inhibition of angiogenesis are VEGF and the VEGF receptors (VEGFRs). Bevacizumab is a recombinant humanised antibody to VEGF that binds to VEGF and thus inhibits activation of VEGFR [37]. Several multitarget kinase inhibitors, which inhibit both angiogenesis and tumour cell growth, are in preclinical and clinical development. These inhibitors were generally identified by screening for activity against a particular kinase, for example VEGFR, and were later found to have activity against other kinases; for example, sunitinib inhibits VEGFR, platelet-derived growth factor receptor (PDGFR), c-KIT and FLT-3 kinase activity [38]; sorafenib (BAY-43-9006) was originally developed as a Raf kinase inhibitor, but also inhibits VEGFR, PDGFR, FLT-3 and c-KIT [39]; dasatinib was identified as a novel inhibitor of both BCR-ABL and SRC kinases, but also targets PDGFR, c-KIT and ephrin A receptors [40].

\subsection{Mechanisms of resistance growth factor receptor/tyrosine kinase-targeted therapies}

Similar to conventional cytotoxic chemotherapies, poor tumour penetration and rapid metabolism/excretion may play a role in resistance to targeted therapies. A number of small-molecule tyrosine kinase inhibitors have been shown to be substrates for drug efflux pumps, including imatinib, which is a substrate for P-gp and BCRP [41,42]. The drug transporter human organic cation transporter 1 (hOCT1) mediates active transport of imatinib into cells, and inhibition of hOCT1 decreases the intracellular concentration of imatinib. Expression of hOCT1 was found to be significantly higher in patients who achieved a complete cytogenetic response to imatinib compared with patients who were > 65\% Philadelphia chromosome-positive after 10 months of treatment $[42,43]$. Gefitinib is a substrate for both BCRP and P-gp and has been shown to modulate drug transport by these efflux pumps in vivo [10,44] and thus may have a role to play in inhibiting chemotherapy drug efflux. It has recently been shown that both erlotinib and lapatinib are also P-gp substrates and similar to gefitinib, they inhibit drug efflux in P-gp-overexpressing cancer cells [45].

Although drug efflux may play a role in resistance to tyrosine kinase inhibitors, alterations in tyrosine kinase signalling in cancer cells, resulting from prolonged exposure to inhibitors, are likely to be the major cause of acquired resistance to tyrosine kinase-targeted therapies [46]. Resistance to inhibition of tyrosine kinase signal transduction in cancer cells may be mediated by: i) alteration of the target tyrosine kinase (e.g., mutations); ii) compensatory signalling from other tyrosine kinases; or iii) alterations in downstream signalling pathways (e.g., PTEN loss resulting in increased Akt activation).

Acquired resistance to kinase inhibition has been most extensively studied with imatinib, which targets BCR-ABL in CML and c-Kit in gastrointestinal stromal tumours and PDGFR- $\alpha$ in hypereosinophilic syndrome. Mutations in the ABL kinase domain, which interfere with binding of imatinib to $\mathrm{ABL}$, are found in $50-90 \%$ of patients (depending on the disease phase) with acquired resistance to imatinib [47]. Analogous mutations have also been observed in KIT and PDGFR- $\alpha$ in association with acquired resistance to treatment [48,49]. BCR-ABL gene amplification can also contribute to imatinib resistance in CML [50].

Mutations in KRAS, resulting in constitutive activation of the Ras/MAPK pathway, have been associated with primary resistance to gefitinib or erlotinib in lung adenocarcinomas [51]. Activating mutations in the tyrosine kinase domain of the EGFR gene are found in $\sim 10 \%$ of NSCLC and are predictive of response to gefitinib and erlotinib in NSCLC patients [36,52]. However, secondary mutations in EGFR can result in the development of resistance. A secondary mutation in exon 20, resulting in a threonine substitution of methionine at position 790, confers resistance to EGFR mutants usually sensitive to either gefitinib or erlotinib [53]. This mutation is analogous to the mutations observed in the kinase domain of $\mathrm{ABL}$ in acquired resistance to imatinib.

Resistance to the EGFR monoclonal antibody, cetuximab, in colorectal cancer has also been associated with KRAS mutations [54]. The mutant EGFR, EGFRvIII, is overexpressed in glioblastoma and head and neck cancer and has been associated with poor response to therapies that target wild-type EGFR. New inhibitors that specifically target EGFRvIII, such as mAb806, may improve response to treatment in cancers that overexpress EGFRvIII [55].

Several mechanisms of resistance to the monoclonal antibody trastuzumab have been proposed [56]. Trastuzumab induces cell cycle arrest in the $G_{1}$ phase, which is mediated by the cyclin-dependent kinase inhibitor p27, and decreased levels of p27 have been associated with resistance to trastuzumab in vitro [57]. Loss of PTEN, a tumour suppressor gene, has also been associated with trastuzumab resistance. PTEN directly inhibits phosphatidyl-inositol 3 kinase and thus decreases the proliferative and antiapoptotic effects of Akt. PTEN activation contributes to the antitumour activity of trastuzumab and PTEN-deficient patients have poorer responses to trastuzumab than patients with normal PTEN [58].

Recent data also suggests that IGF I receptor (IGF-IR) signalling interferes with the growth inhibitory action of trastuzumab in breast cancer cell lines [59]. Nahta et al. [60] 
showed that dimerisation of IGF-IR with HER-2 contributes to trastuzumab resistance in SKBR3-derived trastuzumab-resistant cells. In a study of patients treated with trastuzumab, IGF-IR alone did not correlate with resistance to treatment, but patients with high IGF-IR combined with either high phosphorylated ribosomal S6 protein or low heregulin progressed on trastuzumab treatment [61]. Thus, the exact mechanisms of innate and acquired resistance to trastuzumab treatment are not fully defined at this point. Lapatinib has shown promising results in patients who have progressed on trastuzumab treatment. Therefore, it is possible that resistance to trastuzumab does not confer resistance to inhibition of HER-2 tyrosine kinase activity. BT474 cells selected for resistance to lapatinib showed an increase in estrogen receptor (ER) signalling, thus suggesting a switch in cell survival dependence from HER-2 alone to codependence on ER and HER-2 signalling in acquired resistance to lapatinib [62].

In a study of NSCLC samples, no association was observed between levels of IGF-IR or loss of PTEN and response to gefitinib, thus suggesting they do not play a role in intrinsic resistance to EGFR inhibition by gefitinib in lung cancer [63]. However, Morgillo et al. [64] recently showed that gefitinib induces heterodimerisation of EGFR and IGF-IR in NSCLC cells, resulting in increased survivin levels and protection from gefitinib-induced apoptosis. Therefore, IGF-IR may play a role in acquired resistance to gefitinib in NSCLC. IGF-IR has also been implicated in acquired resistance to gefitinib in breast and prostate cancer cells [65].

Overcoming resistance to small-molecular tyrosine kinase inhibitors may be achieved by using second-generation kinase inhibitors, which retain activity against the mutated targets; for example, novel ABL kinase inhibitors, such as dasatinib, are being developed to overcome imatinib resistance in CML [66]. Similarly, resistance to monoclonal antibody therapies may be achieved by using antibodies that target different conformations of the receptor target, by targeting compensatory signalling pathways or by combining antibody therapies with small-molecule tyrosine kinase inhibitors. However, a greater understanding of the mechanisms of resistance to the individual molecular therapies is required in order to identify the appropriate strategies for overcoming resistance.

\section{Resistance due to stromal factors}

Multiple myeloma (MM), evolving from a highly prevalent premalignant condition termed monoclonal gammopathy of undetermined significance (MGUS) and characterised by an uncontrolled clonal proliferation of malignant plasma cells, is a significant clinical challenge. Recognition of the role of the bone marrow milieu in conferring growth, survival and drug resistance in MM cells, both in laboratory and animal models, has greatly enhanced our understanding and subsequent treatment of this disease [67]. These interactions between MM cell and its microenvironment have been demonstrated using both in in vitro models of MM cells bound to bone marrow stromal cells (BMSCs), as well as in vivo in xenograft models (by injecting fluorochromelabelled MM cells directly into human bone grafts within severe combined immunodeficient mice [SCID-hu]), establishing that adhesion of MM cells to the BMSCs upregulates genes for growth, survival and drug resistance in tumour cells; adhesion molecules on MM and BMSCs; and cytokines in BMSCs [68]. Recently, a genetically based mouse model of human $\mathrm{MM}$ has been generated by overexpression of XBP-1 spliced isoform (XBP-1s), a transcription factor required for plasma cell differentiation that is also highly expressed at a gene and protein level in MM cells versus normal plasma cells [69]. In this model, mice transgenic for Emu-directed (XBP-1s) develop pathogenomic features of MGUS that progressed to $\mathrm{MM}$ with time, providing a unique system for identifying novel targets, validating novel targeted therapies and deciphering mechanisms of resistance. These model systems have allowed for the identification of many promising biologically based therapies to directly or indirectly target the MM and its microenvironment, including thalidomide (CC-5013), bortezomib (PS-341), lenalidomide, VEGFR kinase inhibitor PTK-787, histone deacetylase inhibitors LAQ-824 and SAHA, and LPAAT- $\beta$ inhibitor, in addition to those that specifically target the MM cells (including Hsp-90 inhibitor 17-[allylamino]-17-demethoxygeldanamycin/17-AAG, TRAIL, statins, telomestatin, IGF-1R inhibitor) or bone marrow microenvironment (including I $\mathrm{I} B$ kinase inhibitors and p38MAPK inhibitors [70]).

As a result of advances in oncogenomics increasing our understanding of the role of the bone marrow in the pathogenesis of MM, a new treatment paradigm targeting the tumour cell and its bone marrow microenvironment, by using combinations of these agents to overcome drug resistance and improve patient outcome, is progressing in MM. Efforts are also being invested into the identification of next generation therapies for MM, aligned with oncogenomics to inform the design of combination trials. Examples of promising novel targeted therapies include agents targeting the tumour cell surface (CD40, CS-1, FGFR3), cytokines (VEGF, BAFF) and intracellular targets (MEK, PKC, NF-אB, IKK, cyclin D, proteasomes). Having defined novel agents directed at these targets that can induce cytotoxicity against both MM cells and the bone marrow in both in vitro and in vivo models, intelligent strategies are being applied to define combination therapies to enhance cytotoxicity and overcome drug resistance. For example, microarray profiling has indicated that bortezomib induces Hsp-90 gene transcripts in human MM cells [71], providing the rationale for preclinical functional validation studies showing that combining bortezomib with Hsp-90 inhibitor 17-AAG can enhance cytotoxicity. Two Phase I/II clinical trials of 17-AAG have subsequently defined optimal single-agent dose; an ongoing trial combining bortezomib 
and 17-AAG indicated that the combination can overcome bortezomib resistance; and a Phase III trial comparing bortezomib with bortezomib and $\mathrm{Hsp}-90$ inhibitor is planned. In addition, the emergence of NPI-0052, a proteosome inhibitor that is distinct from bortezomib in structure, mechanism of action and effects on proteasomal activities, overcoming resistance to bortezomib therapies and the heat-shock protein inhibitor KOS-953, indicate the increasing number of treatment options becoming available to the MM patient [72,73].

Despite the very significant therapeutic advances including new drugs and treatment combinations, and thus the cause for optimism for future treatment of MM, MM remains an incurable disease. Future translational research in this disease will focus on further elucidation of mechanism(s) of drug resistance and on the development of molecularly based combination therapies to achieve high frequency and durable responses in the majority of patients.

\section{Proteomic analysis of drug resistance in cancer}

Recent advances in proteome analysis technologies have made it possible to simultaneously identify multiple proteins involved in drug resistance. Expression and functional proteomics are two major areas in cancer research. Expression proteomics seeks to identify proteins/peptides that are differently expressed in tissues. Functional proteomics examines how proteins interact with each other, with DNA/RNA or as components of larger complexes. With recent developments in electrophoresis, imaging, protein labelling, protein array-based approaches and mass spectrometric technologies, along with developments in genomic and protein bioinformatics, the potential to evaluate proteins of interest, including those associated with resistance to specific drugs and those involved in conferring broad resistance to a range of compounds, has greatly improved.

The majority of two-dimensional (2D) electrophoresis analyses of drug resistance focus successfully on drug-resistant cell lines as a model system. Gehrmann et al., [74] examined the protein expression pattern in the cytosol fraction of the adriamycin-resistant MCF-7 cell line (MCF-7/ADR). Protein expression patterns were compared with those of the parental MCF-7 cell line using 2D gel electrophoresis. Some proteins were found to be uniquely expressed in this adriamycin-resistant MCF-7 cell line including annexin I, glutathione- $S$-transferase pi-class and the IL-18 precursor. Shin et al. [75] used a 2D electrophoresis approach for investigating the mechanisms underlying 5-fluorouracil resistance and discovered that ATP synthase downregulation may be a bioenergetic signature of colorectal carcinomas and may also lead to cellular events responsible for 5 -fluorouracil resistance. Tamoxifen is the most frequently used drug for hormone therapy of breast cancer patients, even though a high percentage of women are (or become) refractory to this treatment. Besada et al. [76] examined tamoxifen resistance by electrophoresis and found 12 proteins upregulated in the tamoxifen-resistant line; 9 were downregulated. Many of these proteins may be directly involved in the resistance phenotype.

Unfortunately, 2D electrophoresis remains challenging mainly because of difficulties in the reproducibility of the technique. However, recent improvements in electrophoresis have meant that this traditional methodology has received renewed attention. Two-dimensional difference in-gel electrophoresis (2D-DIGE) is a powerful and widely used method for the analysis of complex protein mixtures extracted from cells, tissues or other biological samples. The fully optimised system (including CyDye fluorescent dyes, imager and DeCyder differential analysis software) offers increased throughput, ease of use, reproducibility and accurate quantitation of protein expression differences. A recent publication by Verrills et al. [77] on intrinsic or acquired resistance to vincristine, an antimicrotubule agent used in the treatment of childhood acute lymphoblastic leukaemia, demonstrated how powerful 2D-DIGE is for the discovery of resistance markers, pharmacoproteomics and signalling pathways in cancer.

Key to the success in analysing drug-resistant models using both traditional 2D electrophoresis and more recently 2D-DIGE is the ability to routinely identify proteins associated with this phenotype. Trypsin-digested proteins can be readily identified using matrix-assisted laser desorption ionisation-time-of-flight mass spectrometry (MALDI-TOF MS), a technique that is both relatively easy to use and reasonably sensitive. MALDI-TOF MS generates a characteristic mass spectrum, known as a peptide mass fingerprint, which is a list of masses for the peptides in a sample. For proteins that are of lower abundance, lower molecular weight or require analysis of post-translational modifications, it is preferable to subject selected peptides to further fragmentation, which can provide the amino acid sequence of the peptide. The amino acid sequence for a specific peptide can be deduced by a process called tandem mass spectrometry (MS/MS).

A recent proteomics technology involved in quantitative analysis of protein mixtures is known as surfaceenhanced laser desorption ionisation-TOF MS (SELDI-TOF MS). This technique uses stainless steel or aluminum-based supports or chips, engineered with chemical (hydrophilic, hydrophobic, preactivated, normal phase, reverse phase, immobilised metal affinity capture and cationic or anionic) or biological (antibody, antigen-binding fragments, DNA, enzyme or receptor) bait surfaces. Masses of proteins ranging from small peptides of $<1000 \mathrm{Da}$ up to proteins of $>300 \mathrm{kDa}$ can be analysed using SELDI-TOF MS. The mass spectrometer produces spectra of complex protein mixtures based on the mass/charge ratio of the proteins in the mixture and their binding affinity to the chip surface. Differentially expressed proteins may be determined from 
these protein profiles by comparing peak intensity. Recent work by Lauten et al. using SELDI-TOF MS identified valosin-containing protein as a putative marker for glucocorticoid resistance in human leukaemia cells [78]. Britten et al. [79] also demonstrated that SELDI-based proteomic profiling may be useful in monitoring the emergence of cisplatin-resistant tumour cell clones.

Isotope-coded affinity tags (ICAT) is a relatively new proteomics technique that allows scientists to examine large numbers of proteins that other technologies are unable to analyse. The ICAT technology involves stable isotope labelling of proteins in a complex mixture, affinity isolation of labelled peptides and amino acid sequence determination and quantification by MS. Stewart et al. [80] employed ICAT coupled with tandem MS to examine the proteomes of cisplatin-sensitive and -resistant ovarian cancer cells. They identified proteins whose expression profiles correlate with cisplatin resistance in ovarian cancer cells.

\section{RNA expression patterns and their manipulation in drug resistance}

An understanding of the mechanisms of drug resistance in cancer is essential so that therapies may be tailored and targeted. One level at which cancer cells may confer resistance is through abnormal expression of specific gene transcripts (mRNAs). To circumvent such resistance, the specific mRNA(s) involved must be identified, so that we can focus on directly manipulating their expression, using technologies such as small-interfering RNA [81], ribozymes [82], antisense [83] or cDNA introduction, as relevant. Clinical material appropriate for investigating the significance of such transcripts is often in short supply and it can be very difficult, for example, to correlate tumour samples, their mRNA expression patterns and the phenotype of resistance they display in vivo. Therefore, studies in cell line models are often conducted in an attempt to model, develop targeted hypotheses and provide an understanding of what may be occurring in the tumours of patients. As such, these studies allow researchers to focus on defined forms of resistance and allow rational studies to be undertaken with human tumours.

Recent development of global microarray techniques for expression analysis of mRNAs has opened the possibility of unbiased global searches for gene expression patterns associated with drug resistance. Using these technologies, many interesting studies aimed at elucidating mechanisms of, and markers for, drug resistance by analysis of cell line models have been reported. Examples of these include an analysis, using cDNA microarrays representing $\sim 8000$ gene transcripts, of the chemoresistance or -sensitivity of 60 human tumour cell lines (NCI60 cell lines) [84,201]. Using a similar approach to study these cell lines, this time by applying Affymetrix oligonucleotide microarrays representing $\sim 6888$ gene transcripts, Staunton et al. [85] identified a panel of transcripts whose expression was predictive of sensitivity or resistance to 232 drugs and showed that clustering of cells based on their gene expression response to drug treatment yielded results distinct to those found with clustering on the basis of drug response. This suggests that we may be able to more accurately predict response by gene expression profiling of tumours rather than simply examining the direct toxicological effect of specific drugs on tumour cells.

Many studies of smaller numbers of cell lines and their resistant variants have also been reported. Analysis of doxorubicin transiently induced and doxorubicin-resistant MCF-7 cells, using cDNA microarrays representing 3801 well annotated transcripts and 1397 expression sequence tags, resulted in the identification of a subset of transcripts common to both variants that are associated with cell cycle, signal transduction, metabolism, ubiquitin proteasome and protein secretion (using RNA from array experiments, validation by reverse transcriptase polymerase chain reaction (RT-PCR) was successful in 58\% of cases) [86. This illustrates there are drug resistance pathways common among tumour cell models, and if extrapolated to human tumours analysis may provide new targets for modulating the impact of resistance.

A number of distinct subtypes of invasive breast carcinomas tumours - namely, luminal A, B and C; normal breast-like; Her-2-overexpressing; and basal-like - have been identified [87]. The basal-like subtype, found in $\sim 20-30 \%$ of patients and observed in BRCA1-related breast cancer, is characterised as triple negative $\left(\mathrm{ER}^{-} / \mathrm{PgR}-/ \mathrm{her}^{-}\right)$, positive for vimentin, EGFR, laminin, fatty acid binding protein 7 (FABP7), cytokeratin 5/6 and 17 expression and is associated with poor prognosis, including early age of onset, high proliferation index and increased incidence of metastasis, together with unresponsiveness to standard chemotherapy, hormone and antibody therapies. Luminal A subtype is characterised by highest expression of ER $\alpha$; luminal B and C, with lower expression of the ER cluster. Luminal tumours have best prognosis, whereas basal-like apparently have the worst outcomes [88]. At present, however, basal and luminal breast tumours are generally treated with the same chemotherapeutic agents. To understand how these tumour types differ in their responses to chemotherapy, a study of the response of two basal (ME16C and HME-CC) and luminal epithelium (MCF-7 and ZR-75-1) to doxorubicin and 5-fluorouracil was performed, using oligonucleotide microarrays, representing 18,861 human genes [89,202]. Results from this study indicated that only two gene transcipts, namely $p 21^{\text {wafl }}$ and chitinase 3 -like 1 , were upregulated in all four cell lines in response to chemotherapy; no mRNAs were downregulated in all four cell lines. Although drugtreated luminal cell lines showed reduced expression of proliferation-associated transcripts (including retinoblastoma 1, ribonucleotide reductase M2, $M C M 4$, chromosome 20 open reading frame 1 and pituitary tumour-transforming 1 ), these were relatively unchanged in basal cell lines. Similarly, XBP1 transcription factor, nucleobindin 1 and heat-shock $70-\mathrm{kDa}$ 
protein 5 were found to be induced in luminal cell lines, but repressed in basal cell lines. Overall, by microarray analysis, treatment with chemotherapeutic drugs was found to repress cell cycle and proliferation-associated mRNAs in luminal cells, but not in basal cell lines. In contrast, many gene transcripts repressed in basal cells were associated with cellular differentiation, including integrin- $\beta 4, C O X-2$, collagen type XII $\alpha 1$ and core promoter element-binding protein. It may be that these differences in mRNA expression patterns are, at least partly, responsible for the diverse outcomes from basal and luminal breast tumours.

Many more microarray studies using cell line models and aimed at identifying gene transcripts associated with the response to chemotherapeutic drugs/drug resistance have been reported. These include a time course cDNA microarray (Altas cancer array, representing 1176 well-characterised genes) analysis of gene transcripts associated with cisplatin resistance in lung cancer cell lines NCI-H226 and NCI-H2170. From this, seven transcripts - namely IFNinducible protein 9-27, MMP11, ERBB3, MIC1, ck19, 40 s ribosomal protein $S 5$ and cytoplasmic $\beta$-actin - were identified as likely to be involved in cisplatin resistance. Analysis of these seven mRNAs, using real-time PCR analysis, indicated a significant correlation with microarray results [90]. Using a cDNA microarray representing 14,081 genes obtained from human gastric cDNA libraries and studying 8 gastric cancer cell lines exposed to 5-fluorouracil, Park et al. [91] reported the identification of 13 gene transcripts directly associated with 5-fluorouracil sensitivity/resistance. Similarly, Hernandez-Vargas et al. [92] reported results from an analysis of breast cancer cell response (using MCF-7 cells) to 5-fluorouracil. This study, using a cDNA microarray representing 6386 different genes, showed overexpression of p53 target genes involved in cell cycle and apoptosis, as well as overexpression of mitochondrial death genes, together with repression of myc expression. Other 'global' expression studies reported include analysis of cisplatin-resistance in oral squamous cell carcinoma (involving a sensitive [Sa-3] and resistant $\mathrm{Sa}-3 \mathrm{R}$ cell line), using a cDNA microarray representing 2201 oral disease origin genes [93]; analysis of paclitaxel- and cisplatin-resistance in ovarian cell lines, a study of 557 human cancer-related cDNA transcripts [94]; and analysis of 10 human tumour cell lines (including myeloma, leukaemia, lymphoma and small-cell lung cancer parental and vincristine, doxorubicin, melphalan and teniposide selected variants) using a cDNA microarray representing 7458 genes [95].

Another application of this technology for identification of gene transcripts associated with drug resistance is through the use of customised microarrays. Using such low-density microarrays, representing 41 gene transporter genes (including 38 ABC transporters, 1 cationic transporter and 2 ATPsensitive potassium channels), Gillet et al. [96] analysed parental and drug-resistant cell line pairs, including T lymphoblastoid leukaemia cells CEM/ADR5000; leukaemia cells HL60/HL60AR; and breast MCF-7/CH1000 cell lines. Results from this study indicated increased expression of expected $\mathrm{ABC}$ transporter gene transcripts, as well as other mRNAs in the drug-resistant variants. These include increased expression of $A B C A 7, A B C B 1 / 4, A B C B 3$, $A B C B 8, A B C B 9, A B C E 1$ and $A B C F 2$ in ADR5000 compared with CEM cells; increased expression of $A B C A 2 / 3, A B C A 4, A B C A 7, A B C B 2, A B C B 3, A B C C 1$, $A B C$ C4, $A B C$ C6/8/9 in HL60AR compared with HL60 cells; and increased expression of $A B C B 6, A B C C 3$, $A B C$ C5, $A B C$ F3, $A B C$ G1 and $A B C$ G2 in $\mathrm{CH} 1000$ cells compared with parental MCF-7 cells.

In addition to studies of cancer cell lines, a number of studies have focused on the analysis of clinical specimens, to identify subtypes of responders versus non-responders. Examples of these studies include a pilot study of 20 oesophageal cases, using a cDNA microarray representing 9216 gene transcripts [97], and reporting the identification of gene transcript signatures predicting outcome from adjuvant chemotherapy. Analysis of 240 (160 test cases, followed by 80 validation cases) untreated diffuse large B-cell lymphoma specimens resulted in the identification of expression patterns associated with improved survival following anthracycline-based chemotherapy [98].

A number of response prediction microarray studies have focused on breast cancer [99]. An analysis of core biopsy specimens from primary breast cancer have identified gene transcript clusters that determine between good and poor responders to neoadjuvant chemotherapy [100]. In a study of 10 cases, Sotiriou et al. [101] analysed linearly amplified RNA from breast fine needle aspirations before and 21 days postchemotherapy (adriamycin plus cyclophosphamide) using a 7600 feature cDNA microarray. From this, 37 genes were reported to be differentially expressed in pretreatment specimens, while Chang et al. [102] studied 24 (11 sensitive and 13 resistant) cases before and post neoadjuvant docetaxel, using the U95Av2 array and reported differential expression of 92 genes correlating with docetaxel response. Studying specimens from a group of 24 patients and using cDNA microarrays to identify transcripts associated with response to sequential treatment of paclitaxel, followed by 5 -fluorouracil, doxorubicin and cyclophosphamide, Ayers et al. [103] identified a 74 gene predictor which, when tested in a further cohort of 18 specimens showed an accuracy of $78 \%$.

\section{Conclusion}

Our initially simple models of drug transport in cancer cells have, of late, become more complex. Physiologically important tissues of the body and cancer cells have complex coordinated uptake and efflux mechanisms with which they can exert significant control over the intracellular concentration of cancer drugs (as well as transcellular drug movement [drug vectoring]). We know that many tumours hijack such mechanisms, thereby reducing the efficacy of chemotherapy. 
The tumour microenvironment, particularly in terms of signalling molecules and other complex stromal signals, can also play a major role in resistance and is potentially a key target of intervention for controlling resistance.

Transcriptomic and proteomic studies of tumour, stromal and cultured cell material have greatly helped our understanding of the significance and contributions both of individual genes/proteins and more general pathways to the underlying mechanism of resistance. Although complex to interpret, taken as a whole these studies suggest that although there can be dominant causes of resistance of the cells in general, there are several different and distinct molecular alterations that contribution to any given resistance phenotype. It appears unlikely that any single gene or protein will act either as a good marker for resistance; rather it is the profile of genotypic and proteomic alterations that may give an appropriate level of prognostic, predictive and diagnostic information.

The ongoing research challenges are:

- To identify the common contributory mechanisms of cancer in order to develop therapeutic strategies and drug combinations that intelligently manage the molecular alterations in cancer, including in particular, a portfolio of regimes that adapt to the molecular evolution of tumours in the body and the accompanying various resistance phenotypes that lead to tumour survival.

- To better characterise the key pathways and dominant contributory factors leading to tumour cel survival.

- To identify specific routes to overcome the resistance response in a cancer-specific manner and thereby deliver an effective cancer-killing payload to the tumour. Modern cell modelling, proteomic and genomic approaches are now gradually coming together with better clinical translation of the implications of such findings to generate novel, rational treatment approaches to the problems of resistance.

\section{Expert opinion}

Resistance to chemotherapeutic agents can be inherent or acquired. The field so far has focused on resistance mechanisms involving overexpression of drug efflux pumps, overexpression of other normal detoxification/protective cellular mechanisms and mutations in molecular targets. In general, approaches to overcoming resistance have included using combinations and/or sequential scheduling agents with different structural and mechanistic properties, and using inhibitors of the protective mechanisms, in particular efflux pump inhibitors. The success of these approaches has been partial rather than spectacular, probably due to a combination of reasons; the multifactional nature of resistance in many cancers, and the narrow window of opportunity in the therapeutic ratio (so that overcoming drug efflux overexpression can also damage normal tissues, for example) may be particularly important.
Resistance mechanisms similar to those discovered for the older cytotoxic agents (e.g., mutated target, drug efflux) have been observed for small-molecule targeted therapeutic agents. There had been some hope that monoclonal antibody therapies might be less susceptible to resistance, as they would not be affected by standard drug detoxification mechanisms, but unfortunately it is now clear that tumours can become insensitive to these therapeutics also. Cells have clearly evolved a wide repertoire of strategies to evade cytotoxic and cytostatic challenges, and the unstable cancer cell genome can readily generate variants capable of exaggerating these responses.

Resistance to chemotherapy can also be a complex phenomenon involving cell-cell and cell-matrix interactions, with stromal and endothelial cells playing key roles in resistance to immunomodulatory agents in, for example, multiple myeloma. More global alterations in cellular pathways involving pro- and antiapoptotic proteins and stress-related (e.g., heat shock) proteins can also play important roles. Global mRNA and proteomic (and more recently microRNA) screening approaches allied to bioinformatics may be powerful tools to discover new mechanisms of resistance, although the masses of data generated can be difficult to interpret definitively.

Although the field is complex, and the results so far are far from perfect, there is reason to be optimistic. First, there is now a steady stream of new targeted agents, with nonoverlapping mechanisms and with relatively milder toxicities, coming through clinical trial. Second, although much remains to be discovered, great progress is being made in identifying and cataloguing what will ultimately be a finite - even if long - list of resistance mechanisms. The goal must be to be able to classify tumours, on an ongoing basis, with respect to the resistance mechanism they express, and to use the growing armamentarian of drugs, in particular the newer targeted agents, in an intelligent sequential fashion, based on ongoing - and ideally minimally invasive - monitoring of the evolution of resistance mechanisms in each patient's tumour. The goal would become, therefore, in those patients where the tumour could not be completely eliminated, to make cancer at least a chronic, episodic disease rather than a terminal one. We suggest that this may be an achievable goal over the next 10 years if the momentum of drug development and resistance mechanism research continues at it current rates Complete cure/elimination as opposed to satisfactory management of cancers that have become resistant and progressed remains an elusive goal, however, and will require some fundamentally new approaches, perhaps at the epigenetic level, which are not yet available to researchers and clinicians in the field.

\section{Declaration of interest}

Research supported by Ireland's HEA PRTLI Cycle 3, Science Foundation Ireland, HRB and Dublin City University's Research Fellowship. 


\section{Bibliography}

1. DEAN M, FOJO T, BATES S: Tumour stem cells and drug resistance. Nat. Rev. Cancer (2005) 5(4):275-284.

2. CLEARY I, DOHERTY G, MORAN E, CLYNES M: The multidrug-resistant human lung tumour cell line, DLKP-A10, expresses novel drug accumulation and sequestration systems. Biochem. Pharmacol. (1997) 53(10):1493-1502.

3. STEINER E, HOLZMANN K, ELBLING L, MICKSCHE M, BERGER W: Cellular functions of vaults and their involvement in multidrug resistance. Curr. Drug Targets (2006) 7(8):923-934

4. CHEN KG, VALENCIA JC, LAI B et al: Melanosomal sequestration of cytotoxic drugs contributes to the intractability of malignant melanomas. Proc. Natl. Acad. Sci. USA (2006) 103(26):9903-9907.

5. HEENAN M, O'DRISCOLL L, CLEARY I, CONNOLLY L, CLYNES M: Isolation from a human MDR lung cell line of multiple clonal subpopulations which exhibit significantly different drug resistance. Int. J. Cancer (1997) 71(5):907-915.

6. O'CONNOR R, O'LEARY M, BALLOT J et al: : A Phase I clinical and pharmacokinetic study of the multi-drug resistance protein-1 (MRP-1) inhibitor sulindac, in combination with epirubicin in patients with advanced cancer. Cancer Chemother. Pharmacol. (2007) 59(1):79-87.

7. LEONARD GD, POLGAR O, BATES SE: $\mathrm{ABC}$ transporters and inhibitors: new targets, new agents. Curr. Opin. Investig. Drugs (2002) 3(11):1652-1659.

8. JULIANO RL, LING V: A surface glycoprotein modulating drug permeability in Chinese hamster ovary cell mutants. Biochim. Biophys. Acta (1976) 455(1):152-162.

9. CALLAGHAN R, FORD RC, KERR ID: The translocation mechanism of P-glycoprotein. FEBS Lett. (2006) 580(4):1056-1063.

10. OZVEGY-LACZKA C, CSEREPES J, ELKIND NB, SARKADI B: Tyrosine kinase inhibitor resistance in cancer: role of $\mathrm{ABC}$ multidrug transporters. Drug Resist. Updat. (2005) 8(1-2):15-26.

11. COLE SP, BHARDWAJ G, GERLACH JH et al.: Overexpression of a transporter gene in a multidrug-resistant human lung cancer cell line. Science (1992) 258(5088):1650-1654.

12. SARKADI B, OZVEGY-LACZKA C, NEMET K, VARADI A: ABCG2 - a transporter for all seasons. FEBS Lett. (2004) 567(1):116-120.

13. KRUH GD, GUO Y, HOPPER-BORGE E, BELINSKY MG, CHEN ZS: ABCC10, ABCC11, and ABCC12. Pflugers Arch. (2007) 453(5):675-684.

14. BORST P, DE WOLF C, VAN DE WETERING K: Multidrug resistance-associated proteins 3, 4, and 5. Pflugers Arch. (2007) 453(5):661-673.

15. TUSNADY GE, SARKADI B, SIMON I, VARADI A: Membrane topology of human ABC proteins. FEBS Lett. (2006) 580(4):1017-1022.

16. AMBUDKAR SV, KIMCHI-SARFATY C, SAUNA ZE, GOTTESMAN MM: P-glycoprotein: from genomics to mechanism. Oncogene (2003) 22(47):7468-7485.

17. COLE SP, DEELEY RG: Transport of glutathione and glutathione conjugates by MRP1. Trends Pharmacol. Sci. (2006) 27(8):438-446.

18. LEONESSA F, CLARKE R: ATP binding cassette transporters and drug resistance in breast cancer. Endocr. Relat. Cancer (2003) 10(1):43-73.

19. PEREZ-TOMAS R: Multidrug resistance: retrospect and prospects in anti-cancer drug treatment. Curr. Med. Chem. (2006) 13(16):1859-1876.

20. STEIN WD, BATES SE, FOJO T: Intractable cancers: the many faces of multidrug resistance and the many targets it presents for therapeutic attack. Curr. Drug Targets (2004) 5(4):333-346.

21. BAKER EK, JOHNSTONE RW, ZALCBERG JR, EL-OSTA A: Epigenetic changes to the MDR1 locus in response to chemotherapeutic drugs. Oncogene (2005) 24(54):8061-8075.

22. LEONARD GD, FOJO T, BATES SE: The role of $A B C$ transporters in clinical practice. Oncologist (2003) 8(5):411-424.

23. PUSZTAI L, WAGNER P, IBRAHIM N et al: : Phase II study of tariquidar, a selective P-glycoprotein inhibitor, in patients with chemotherapy-resistant, advanced breast carcinoma. Cancer (2005) 104(4):682-691.
24. CLARKE R, LEONESSA F, TROCK B: Multidrug resistance/P-glycoprotein and breast cancer: review and meta-analysis. Semin. Oncol. (2005) 32(6 Suppl. 7):S9-S15.

25. O'CONNOR R: The pharmacology of cancer resistance. Anticancer Res. (2007) 27(3A):1267-1272.

26. KIM RB: Organic anion-transporting polypeptide (OATP) transporter family and drug disposition. Eur. J. Clin. Invest. (2003) 33(Suppl. 2):1-5.

27. SMITH NF, ACHARYA MR, DESAI N, FIGG WD, SPARREBOOM A: Identification of OATP1B3 as a high-affinity hepatocellular transporter of paclitaxel. Cancer Biol. Ther. (2005) 4(8):815-818.

28. KAPP T, MULLER S, GUST R: Dinuclear alkylamine platinum(II) complexes of [1,2-bis(4-fluorophenyl)ethylenediamine] platinum(II): influence of endocytosis and copper and organic cation transport systems on cellular uptake. ChemMedChem (2006) 1(5):560-564

29. SU Y, ZHANG X, SINKO PJ: Human organic anion-transporting polypeptide OATP-A (SLC21A3) acts in concert with P-glycoprotein and multidrug resistance protein 2 in the vectorial transport of Saquinavir in Hep G2 cells. Mol. Pharm. (2004) 1(1):49-56.

30. CUI Y, KONIG J, NIES AT et al: Detection of the human organic anion transporters SLC21A6 (OATP2) and SLC21A8 (OATP8) in liver and hepatocellular carcinoma. Lab. Invest. (2003) 83(4):527-538

31. O'DWYER ME, MAURO MJ, DRUKER BJ: STI571 as a targeted therapy for CML. Cancer Invest. (2003) 21(3):429-438.

32. SLAMON DJ, CLARK GM, WONG SG, LEVIN WJ, ULLRICH A, MCGUIRE WL: Human breast cancer: correlation of relapse and survival with amplification of the HER-2/neu oncogene. Science (1987) 235(4785):177-182.

33. DEMONTY G, BERNARD-MARTY C, PUGLISI F, MANCINI I, PICCART M: Progress and new standards of care in the management of HER-2 positive breast cancer. Eur. J. Cancer (2007) 43(3):497-509.

34. GEYER CE, FORSTER J, LINDQUIST D et al.: Lapatinib plus capecitabine for HER2-positive 
advanced breast cancer. N. Engl. J. Med. (2006) 355(26):2733-2743.

35. LYNCH TJ, BELL DW, SORDELLA R et al: : Activating mutations in the epidermal growth factor receptor underlying responsiveness of non-small-cell lung cancer to gefitinib. N. Engl. J. Med. (2004) 350(21):2129-2139.

36. PAEZ JG, JANNE PA, LEE JC et al: EGFR mutations in lung cancer: correlation with clinical response to gefitinib therapy. Science (2004) 304(5676):1497-1500.

37. RANIERI G, PATRUNO R, RUGGIERI E, MONTEMURRO S, VALERIO P, RIBATTI D: Vascular endothelial growth factor (VEGF) as a target of bevacizumab in cancer: from the biology to the clinic. Curr. Med. Chem. (2006) 13(16):1845-1857.

38. MENDEL DB, LAIRD AD, XIN X et al: In vivo antitumor activity of SU11248, a novel tyrosine kinase inhibitor targeting vascular endothelial growth factor and platelet-derived growth factor receptors: determination of a pharmacokinetic/pharmacodynamic relationship. Clin. Cancer Res. (2003) 9(1):327-337.

39. WILHELM SM, CARTER C, TANG L et al.: BAY 43-9006 exhibits broad spectrum oral antitumor activity and targets the RAF/MEK/ERK pathway and receptor tyrosine kinases involved in tumor progression and angiogenesis. Cancer Res. (2004) 64(19):7099-7109.

40. LOMBARDO LJ, LEE FY, CHEN P et al: Discovery of $N$-(2-chloro-6-methylphenyl)-2-(6-(4-(2-hydroxyethyl)piperazin-1-yl)-2-methylpyrimidin4-ylamino)thiazole-5-carboxamide (BMS-354825), a dual Src/Abl kinase inhibitor with potent antitumor activity in preclinical assays. J. Med. Chem. (2004) 47(27):6658-6661.

41. BURGER $H$, VAN TOL $H, B R O K M$ et al: Chronic imatinib mesylate exposure leads to reduced intracellular drug accumulation by induction of the ABCG2 (BCRP) and ABCB1 (MDR1) drug transport pumps. Cancer Biol. Ther. (2005) 4(7):747-752.

42. THOMAS J, WANG L, CLARK RE, PIRMOHAMED M: Active transport of imatinib into and out of cells: implications for drug resistance. Blood (2004) 104(12):3739-3745.

43. CROSSMAN LC, DRUKER BJ, DEININGER MW, PIRMOHAMED M,
WANG L, CLARK RE: hOCT 1 and resistance to imatinib. Blood (2005) 106(3):1133-1134.

44. LEGGAS M, PANETTA JC, ZHUANG Y et al.: Gefitinib modulates the function of multiple ATP-binding cassette transporters in vivo. Cancer Res. (2006) 66(9):4802-4807.

45. COLLINS D, O'CONNOR R, O'DONOVAN N, CLYNES M, CROWN J: Lapatinib inhibits P-glycoprotein (P-gp) and synergistically enhances taxane cytotoxicity in $\mathrm{P}_{-\mathrm{gp}^{+}}$cell lines. Ann. Oncol. (2006) 17(S9):57.

46. VILORIA-PETIT AM, KERBEL RS: Acquired resistance to EGFR inhibitors: mechanisms and prevention strategies. Int. J. Radiat. Oncol. Biol. Phys. (2004) 58(3):914-926.

47. DEININGER M, BUCHDUNGER E, DRUKER BJ: The development of imatinib as a therapeutic agent for chronic myeloid leukemia. Blood (2005) 105(7):2640-2653.

48. HEINRICH MC, CORLESS CL, BLANKE CD et al: : Molecular correlates of imatinib resistance in gastrointestinal stromal tumors. J. Clin. Oncol. (2006) 24(29):4764-4774.

49. COOLS J, DEANGELO DJ, GOTLIB J et al: : A tyrosine kinase created by fusion of the PDGFRA and FIP1L1 genes as a therapeutic target of imatinib in idiopathic hypereosinophilic syndrome. N. Engl. J. Med. (2003) 348(13):1201-1214.

50. GADZICKI D, VON NEUHOFF N, STEINEMANN D et al:: $B C R-A B L$ gene amplification and overexpression in a patient with chronic myeloid leukemia treated with imatinib. Cancer Genet. Cytogenet. (2005) 159(2):164-167.

51. PAO W, WANG TY, RIELY GJ et al: KRAS mutations and primary resistance of lung adenocarcinomas to gefitinib or erlotinib. PLoS Med. (2005) 2(1):E17.

52. PAO W, MILLER V, ZAKOWSKI M et al: : EGF receptor gene mutations are common in lung cancers from "never smokers" and are associated with sensitivity of tumors to gefitinib and erlotinib. Proc. Natl. Acad. Sci. USA (2004) 101(36):13306-13311.

53. PAO W, MILLER VA, POLITI KA et al: : Acquired resistance of lung adenocarcinomas to gefitinib or erlotinib is associated with a second mutation in the EGFR kinase domain. PLoS Med. (2005) 2(3):E73.

54. LIEVRE A, BACHET JB, LE CORRE D et al: KRAS mutation status is predictive of response to cetuximab therapy in colorectal cancer. Cancer Res. (2006) 66(8):3992-3995.

55. PERERA RM, NARITA Y, FURNARI FB et al.: Treatment of human tumor xenografts with monoclonal antibody 806 in combination with a prototypical epidermal growth factor receptor-specific antibody generates enhanced antitumor activity. Clin. Cancer Res. (2005) 11(17):6390-6399.

56. NAHTA R, ESTEVA FJ: Herceptin: mechanisms of action and resistance. Cancer Lett. (2006) 232(2):123-138.

57. NAHTA R, TAKAHASHI T, UENO NT, HUNG MC, ESTEVA FJ: P27(kip1) down-regulation is associated with trastuzumab resistance in breast cancer cells. Cancer Res. (2004) 64(11):3981-3986.

58. NAGATA Y, LAN KH, ZHOU X et al: PTEN activation contributes to tumor inhibition by trastuzumab, and loss of PTEN predicts trastuzumab resistance in patients. Cancer Cell (2004) 6(2):117-127.

59. LU Y, ZI X, POLLAK M: Molecular mechanisms underlying IGF-I-induced attenuation of the growth-inhibitory activity of trastuzumab (Herceptin) on SKBR3 breast cancer cells. Int. J. Cancer (2004) 108(3):334-341.

60. NAHTA R, YUAN LX, ZHANG B, KOBAYASHI R, ESTEVA FJ: Insulin-like growth factor-I receptor/human epidermal growth factor receptor 2 heterodimerization contributes to trastuzumab resistance of breast cancer cells. Cancer Res. (2005) 65(23):11118-11128.

61. SMITH BL, CHIN D, MALTZMAN W, CROSBY K, HORTOBAGYI GN, BACUS SS: The efficacy of herceptin therapies is influenced by the expression of other erbB receptors, their ligands and the activation of downstream signalling proteins. Br. J. Cancer (2004) 91(6):1190-1194.

62. XIA W, BACUS S, HEGDE P et al:: A model of acquired autoresistance to a potent ErbB2 tyrosine kinase inhibitor and a therapeutic strategy to prevent its onset in breast cancer. Proc. Natl. Acad. Sci. USA (2006) 103(20):7795-7800. 
63. CAPPUZZO F, TOSCHI L, TALLINI G et al:: Insulin-like growth factor receptor 1 (IGFR-1) is significantly associated with longer survival in non-small-cell lung cancer patients treated with gefitinib. Ann. Oncol. (2006) 17(7):1120-1127.

64. MORGILLO F, KIM WY, KIM ES, CIARDIELLO F, HONG WK, LEE HY: Implication of the insulin-like growth factor-IR pathway in the resistance of non-small cell lung cancer cells to treatment with gefitinib. Clin. Cancer Res. (2007) 13(9):2795-2803.

65. JONES HE, GODDARD L, GEE JM et al: Insulin-like growth factor-I receptor signalling and acquired resistance to gefitinib (ZD1839; Iressa) in human breast and prostate cancer cells. Endocr. Relat. Cancer (2004) 11(4):793-814.

66. SHAH NP, TRAN C, LEE FY, CHEN P, NORRIS D, SAWYERS CL: Overriding imatinib resistance with a novel ABL kinase inhibitor. Science (2004) 305(5682):399-401.

67. HWANG JJ, GHOBRIAL IM, ANDERSON KC: New frontiers in the treatment of multiple myeloma. Scientific WorldJournal (2006) 6:1475-1503.

68. MITSIADES CS, MITSIADES NS, MUNSHI NC, RICHARDSON PG, ANDERSON KC: The role of the bone microenvironment in the pathophysiology and therapeutic management of multiple myeloma: interplay of growth factors, their receptors and stromal interactions. Eur. J. Cancer (2006) 42(11):1564-1573.

69. CARRASCO DR, SUKHDEO K, PROTOPOPOVA M et al:: The differentiation and stress response factor XBP-1 drives multiple myeloma pathogenesis. Cancer Cell (2007) 11(4):349-360.

70. HIDESHIMA T, BERGSAGEL PL, KUEHL WM, ANDERSON KC: Advances in biology of multiple myeloma: clinical applications. Blood (2004) 104(3):607-618.

71. MULLIGAN G, MITSIADES C, BRYANT B et al:: Gene expression profiling and correlation with outcome in clinical trials of the proteasome inhibitor bortezomib. Blood (2007) 109(8):3177-3188.

72. CHAUHAN D, HIDESHIMA T, ANDERSON KC: A novel proteasome inhibitor NPI-0052 as an anticancer therapy. Br. J. Cancer (2006) 95(8):961-965.

73. ANDERSON KC: Targeted therapy of multiple myeloma based upon tumor-microenvironmental interactions. Exp. Hematol. (2007) 35(4 Suppl. 1):155-162.

74. GEHRMANN ML, FENSELAU C, HATHOUT Y: Highly altered protein expression profile in the adriamycin resistant MCF-7 cell line. J. Proteome Res. (2004) 3(3):403-409.

75. SHIN YK, YOO BC, CHANG HJ et al.: Down-regulation of mitochondrial F1F0-ATP synthase in human colon cancer cells with induced 5-fluorouracil resistance. Cancer Res. (2005) 65(8):3162-3170.

76. BESADA V, DIAZ M, BECKER M, RAMOS Y, CASTELLANOS-SERRA L, FICHTNER I: Proteomics of xenografted human breast cancer indicates novel targets related to tamoxifen resistance. Proteomics (2006) 6(3):1038-1048.

77. VERRILLS NM, LIEM NL, LIAW TY, HOOD BD, LOCK RB, KAVALLARIS M: Proteomic analysis reveals a novel role for the actin cytoskeleton in vincristine resistant childhood leukaemia - an in vivo study. Proteomics (2006) 6(5):1681-1694.

78. LAUTEN M, SCHRAUDER A, KARDINAL C et al.: Unsupervised proteome analysis of human leukaemia cells identifies the valosin-containing protein as a putative marker for glucocorticoid resistance. Leukemia (2006) 20(5):820-826.

79. BRITTEN RA, HARDY C, VLAHOU A, GREGORY B, GIRI PS, DRAKE R: Identification of reproducible low mass SELDI protein profiles specific to cisplatin resistance in human ovarian cancer cells. Oncol. Rep. (2005) 14(5):1323-1330.

80. STEWART JJ, WHITE JT, YAN X et al: Proteins associated with cisplatin resistance in ovarian cancer cells identified by quantitative proteomic technology and integrated with mRNA expression levels. Mol. Cell Proteomics (2006) 5(3):433-443.

81. JOYCE H, BRAY I, CLYNES M: RNA interference with SiRNA. Cancer Genomics Proteomics (2006) 3:127-136.

82. KHAN AU: Ribozyme: a clinical tool. Clin. Chim. Acta (2006) 367(1-2):20-27.

83. WACHECK V, ZANGEMEISTER-WITTKE U:
Antisense molecules for targeted cancer therapy. Crit. Rev. Oncol. Hematol. (2006) 59(1):65-73.

84. SCHERF U, ROSS DT, WALTHAM M et al:: A gene expression database for the molecular pharmacology of cancer. Nat. Genet. (2000) 24(3):236-244.

85. STAUNTON JE, SLONIM DK, COLLER HA et al: Chemosensitivity prediction by transcriptional profiling. Proc. Natl. Acad. Sci. USA (2001) 98(19):10787-10792.

86. KUDOH K, RAMANNA M, RAVATN R et al: : Monitoring the expression profiles of doxorubicin-induced and doxorubicin-resistant cancer cells by cDNA microarray. Cancer Res. (2000) 60(15):4161-4166

87. SORLIE T, PEROU CM, TIBSHIRANI R et al: : Gene expression patterns of breast carcinomas distinguish tumor subclasses with clinical implications. Proc. Natl. Acad. Sci. USA (2001) 98(19):10869-10874.

88. KAKLAMANI VG, GRADISHAR WJ: Gene expression in breast cancer. Curr. Treat. Options Oncol. (2006) 7(2):123-128.

89. TROESTER MA, HOADLEY KA, SORLIE T et al.: Cell-type-specific responses to chemotherapeutics in breast cancer. Cancer Res. (2004) 64(12):4218-4226.

90. WHITESIDE MA, CHEN DT, DESMOND RA, ABDULKADIR SA, JOHANNING GL: A novel time-course cDNA microarray analysis method identifies genes associated with the development of cisplatin resistance. Oncogene (2004) 23(3):744-752.

91. PARK JS, YOUNG YOON S, KIM JM, YEOM YI, KIM YS, KIM NS: Identification of novel genes associated with the response to 5-FU treatment in gastric cancer cell lines using a cDNA microarray. Cancer Lett. (2004) 214(1):19-33.

92. HERNANDEZ-VARGAS $\mathrm{H}$, BALLESTAR E, CARMONA-SAEZ P et al:: Transcriptional profiling of MCF7 breast cancer cells in response to 5-fluorouracil: relationship with cell cycle changes and apoptosis, and identification of novel targets of $\mathrm{p} 53$. Int. J. Cancer (2006) 119(5):1164-1175.

93. NAKATANI K, NAKAMURA M, UZAWA K et al.: Establishment and gene analysis of a cisplatin-resistant cell line, 
Sa-3R, derived from oral squamous cell carcinoma. Oncol. Rep. (2005)

13(4):709-714.

94. GOTO T, TAKANO M, SAKAMOTO M

et al: Gene expression profiles with cDNA microarray reveal RhoGDI as a predictive marker for paclitaxel resistance in ovarian cancers. Oncol. Rep. (2006) 15(5):1265-1271.

95. RICKARDSON L, FRYKNAS M, DHAR $S$ et al: : Identification of molecular mechanisms for cellular drug resistance by combining drug activity and gene expression profiles. Br. J. Cancer (2005) 93(4):483-492.

96. GILLET JP, EFFERTH T, STEINBACH D et al:: Microarray-based detection of multidrug resistance in human tumor cells by expression profiling of ATP-binding cassette transporter genes. Cancer Res. (2004) 64(24):8987-8993.

97. KIHARA C, TSUNODA T, TANAKA T et al: : Prediction of sensitivity of esophageal tumors to adjuvant chemotherapy by cDNA microarray analysis of gene-expression profiles. Cancer Res. (2001) 61(17):6474-6479.
98. ROSENWALD A, WRIGHT G, CHAN WC et al: The use of molecular profiling to predict survival after chemotherapy for diffuse large-B-cell lymphoma. N. Engl. J. Med. (2002) 346(25):1937-1947.

99. REIS-FILHO JS, WESTBURY C, PIERGA JY: The impact of expression profiling on prognostic and predictive testing in breast cancer. J. Clin. Pathol. (2006) 59(3):225-231.

100. BUCHHOLZ TA, STIVERS DN, STEC J et al:: Global gene expression changes during neoadjuvant chemotherapy for human breast cancer. Cancer J. (2002) 8(6):461-468.

101. SOTIRIOU C, POWLES TJ, DOWSETT M et al:: Gene expression profiles derived from fine needle aspiration correlate with response to systemic chemotherapy in breast cancer. Breast Cancer Res. (2002) 4(3):R3.

102. CHANG JC, WOOTEN EC, TSIMELZON A et al: Gene expression profiling for the prediction of therapeutic response to docetaxel in patients with breast cancer. Lancet (2003) 362(9381):362-369.
103. AYERS M, SYMMANS WF, STEC J et al:: Gene expression profiles predict complete pathologic response to neoadjuvant paclitaxel and fluorouracil, doxorubicin, and cyclophosphamide chemotherapy in breast cancer. J. Clin. Oncol. (2004) 22(12):2284-2293.

\section{Websites}

201. http://www.discover.nci.nih.gov

202. http://www.labonweb.com/chips/ libraries.html

\section{Affiliation}

Robert O'Connor PhD, Martin Clynes ${ }^{\dagger} \mathrm{PhD}$, Paul Dowling PhD, Norma O’Donovan PhD \& Lorraine O’Driscoll PhD

${ }^{\dagger}$ Author for correspondence Dublin City University,

National Institute for Cellular

Biotechnology (NICB),

Dublin 9, Ireland

Tel: +353 1700 5700; Fax: +353 1700 5484;

E-mail: martin.clynes@dcu.ie 


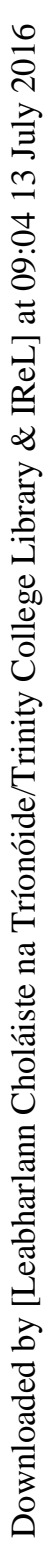

